\title{
Collateral sprouting of nociceptive C-fibers after cut or capsaicin treatment of the sciatic nerve in adult rats
}

\author{
Antti Pertovaara \\ Department of Physiology, University of Michigan, and Neurology Reseach Laboratories \\ (Neurophysiology), Veterans Administration Medical Center, Ann Arbor, MI (U.S.A.)
}

(Received 4 March 1988; Revised version received 11 April 1988; Accepted 11 April 1988)

Key words: Nerve injury; Sprouting; Unmyelinated fiber; Capsaicin; Peripheral nerve: Nociception

The innervation area of nociceptive C-fibers in the saphenous nerve of adult rats was detected by the Evans blue technique, in which antidromic excitation of the nociceptive C-fibers causes a visible extravasation of dye in the skin it supplied. One month after cutting the sciatic nerve, the nociceptive $C$-fibers of the intact saphenous nerve had sprouted to the sciatic area. When capsaicin was locally applied to the nerve trunk to destroy C-fibers of the sciatic nerve, there was no sprouting of nociceptive C-fibers from the saphenous nerve. Thus, myelinated fibers and/or the functionally impaired C-fibers of the sciatic nerve are enough to prevent collateral sprouting of nociceptive $\mathrm{C}$-fibers from the saphenous nerve.

There is both clinical $[7,9,14,17]$ and experimental $[1,3,4,13,19]$ evidence indicating that after lesion of a cutaneous sensory nerve the sensory nerve fibers of the neighbouring intact nerve may expand to the area previously innervated by the lesioned nerve. Only thin myelinated $A \delta$ fibers [3] and unmyelinated C-fibers [1,4] sprout but not the thick mechanoreceptive A $\beta$ fibers [8].

The aim of the current study was to find out whether one needs to lesion the whole sciatic nerve to induce collateral sprouting in nociceptive $\mathrm{C}$-fibers of the saphenous nerve or whether a selective lesioning of the sciatic $\mathrm{C}$-fibers will induce nociceptive $\mathrm{C}$-fiber sprouting in the saphenous nerve. The innervation area of the nociceptive $\mathrm{C}$ fibers can be revealed by a dye-labelled plasma extravasation technique; it has been demonstrated that antidromic activation of nociceptive $C$-fibers produces plasma extravasation, whereas antidromic activation of other sensory nerves is in this respect ineffective [12]. Capsaicin [2, 5] was used to produce a selective lesion of sciatic $C$ fibers.

Adult male Sprague-Dawley rats were used in the experiments. At the time of

Correspondence: A. Pertovaara. Permanent address: Department of Physiology, University of Helsinki, Siltavuorenpenger $20 \mathrm{~J}, 00170$ Helsinki, Finland. 
initial operation they had a body weight in the range $250-350 \mathrm{~g}$. In the preparatory operation the animals were anesthetized using chloral hydrate $(400 \mathrm{mg} / \mathrm{kg}$ i.p.). The right sciatic nerve was exposed in the thigh. For one group of rats a $0.5-\mathrm{cm}$ piece of the sciatic nerve was cut away and the proximal stump was ligated with silk to prevent regeneration. For one group of rats a cotton ball soaked in capsaicin (Fluka, $1.5 \%$ in mineral oil) was placed on the exposed sciatic nerve for $15 \mathrm{~min}$ after which the extra capsaicin was washed away. The wounds were closed with catgut, penicillin was administered and the animals were allowed to recover.

One month after the initial operation the animals were reanesthetized as before. The right saphenous nerve was exposed and covered with warm mineral oil in a pool formed from the skin flaps. The cut distal end of the nerve was placed on a pair of platinum-iridium electrodes. Evans blue in a dose of $50 \mathrm{mg} / \mathrm{kg}$ was then administered i.v. as a $50-\mathrm{mg} / \mathrm{ml}$ solution in $0.9 \%$ saline. The saphenous nerve was stimulated at $10 \mathrm{~Hz}$ for $10 \mathrm{~min}$ at $\mathrm{C}$-fiber intensity ( $5 \mathrm{~mA}, 0.5 \mathrm{~ms}$ duration). In the capsaicin-treated group the sciatic nerve was also antidromically stimulated (central to the site of the capsaicin application) as above to verify the effect of capsaicin on nociceptive $C$ fibers. The sciatic nerve stimulation was done after the effect of saphenous nerve stimulation had been evaluated.

The extent of the blue colouration of the skin was mapped on scale diagrams of the foot as described earlier by Brenan [1]. Fig. la shows the sharpness of the boundary of the coloured area. The diagrams represented the skin of the whole foot up to a line $43 \mathrm{~mm}$ from the tip of digit III. Areas were measured from the diagrams using computerised graphic systems. Control experiments were carried out on separ-
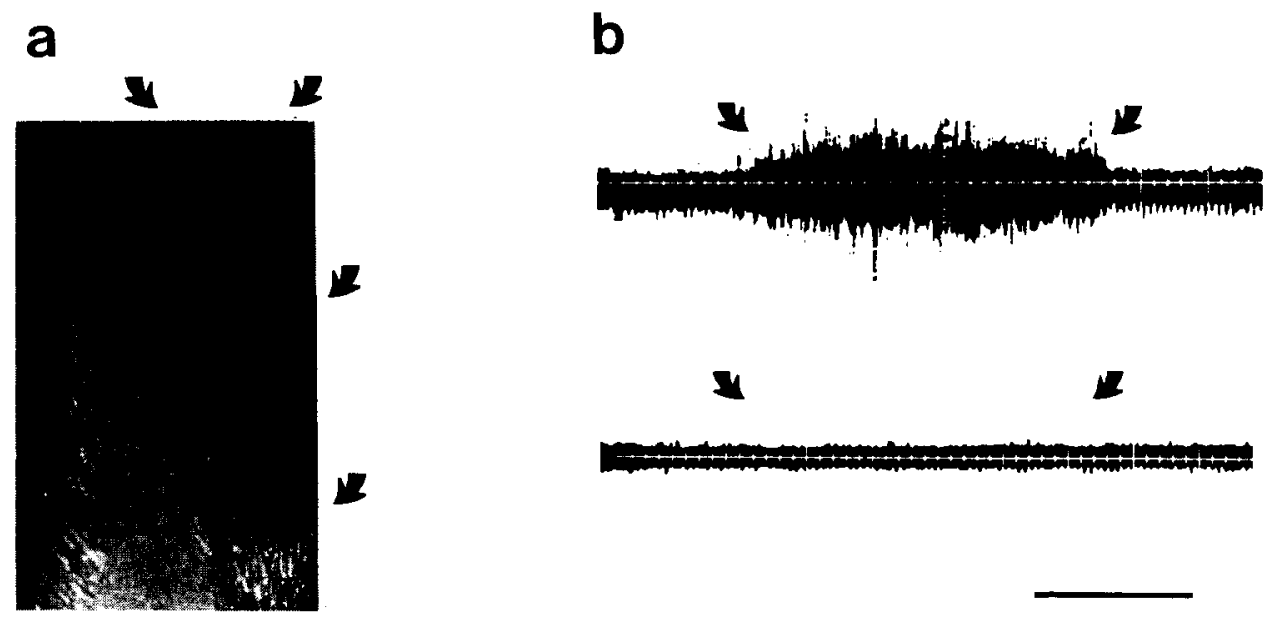

Fig. 1. a: a photographic example of extravasation in the skin of the rat hindlimb demonstrating the sharpness of the boundary of the extravasation area. The dark area (between the arrows) indicates extravasation. The scale is in $\mathrm{mm}$. b: photographic examples of multifiber activity in the saphenous nerve. The stimulus (a skin scratch with a needle at nociceptive intensity) was applied between the arrows. In the lower trace the stimulus was applied to the light area in a (outside the extravasation area), and in the upper trace the stimulus was applied to the dark area (the area of extravasation) in a. Bar $=0.5 \mathrm{~s}$. 
ate animals without nerve injury. Within-animal controls were not used in case the selective or whole-nerve lesion produced effects on the contralateral side. Student's $t$-test was used for statistical comparisons.

The original size of each group was 6 rats. However, in the sciatic cut group two of the rats had autotomized (self-mutilated) part of their right feet which made it impossible to obtain comparable results from these two rats. Thus, two extra rats had to be included in the sciatic cut group to obtain reliable data from 6 rats. None of the normal controls or the capsaicin-treated animals showed any autotomy.

In 3 rats of each group an additional electrophysiological mapping of innervation area was made by recording multi-unit activity of the saphenous nerve. After the completion of the above extravasation experiments, the saphenous nerve was recorded on a platinum-iridium hook electrode. The receptive field was mapped by scratching the skin with a needle. Fig. $1 \mathrm{~b}$ gives an example of multi-unit recordings. Special attention was paid to comparison of electrophysiologically determined boundaries to the boundaries produced by the extravasation technique.

In unoperated control animals the blue area was limited to the medial aspect of the dorsal surface of the limb with no plantar representation (Fig. 2). On average $36.8 \pm 2 \%$ (S.E.M., $n=6$ ) of the dorsal surface was covered by the blue colouration in the controls.

One month after sciatic nerve cut the mean area demarcated by the blue colouration after antidromic stimulation of the saphenous nerve was increased to $60.3 \pm 4 \%$ $(n=6)$, which is significantly larger area than in the control group $(P<0.05)$. The expansion took place only in the dorsal surface (Fig. 2) and no blue colouration was seen in the plantar side in any of the animals.

CONTROL

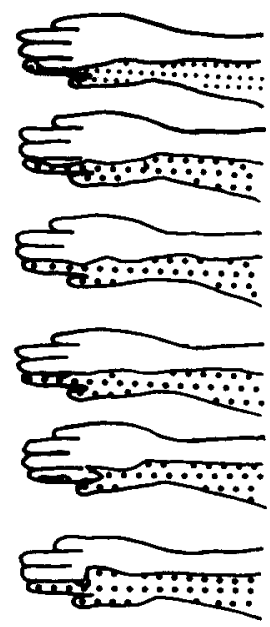

SCIATIC CUT

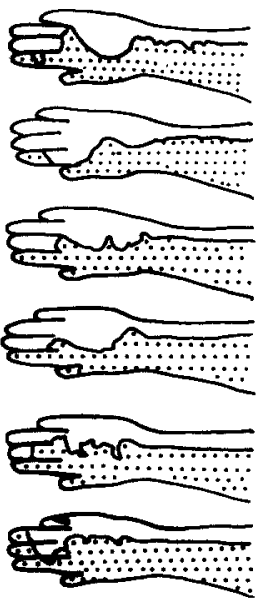

CAPSAICIN

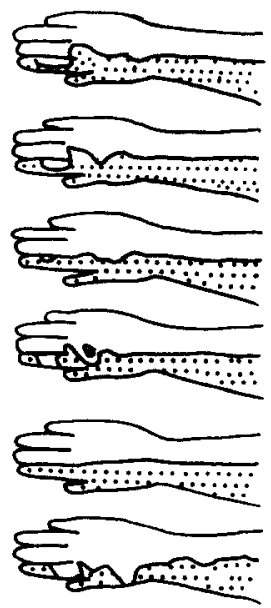

Fig. 2. Extravasation (indicating innervation area of nociceptive $C$-fibers) in dorsal surfaces of the feet of the right hind limbs after antidromic stimulation of the saphenous nerve in different experimental groups. The dotted areas indicate plasma extravasation. 
In the capsaicin group two extra rats were used to test whether there would be an impairment of the extravasation produced by sciatic stimulation $15 \mathrm{~min}$ after the end of capsaicin application. Antidromic stimulation of the sciatic nerve at C-fiber intensity (stimulus site central to the capsaicin application site) produced a vigorous motor response but produced no blue colouration $15 \mathrm{~min}$ after the end of the capsaicin application. Antidromic stimulation of the saphenous nerve in the same limb produced a blue area of equal size as in controls. Also, antidromic activation of the contralateral sciatic nerve produced a well demarcated blue colouration in the whole plantar area and in the lateral side of the dorsal surface of the leg.

One month after the application of capsaicin to the sciatic nerve the blue area produced by antidromic stimulation of the saphenous nerve was $35.0 \pm 2 \%(n=6)$ which is significantly less than in the sciatic nerve cut group $(P<0.05)$ but not different from that in the control group. In all capsaicin-treated animals the blue colouration was restricted to the dorsal surface of the leg (Fig. 2). One month after capsaicin treatment the antidromic stimulation of the treated sciatic nerve produced a vigorous motor response in all animals but no blue colouration in the sciatic area, except a few small spots in 3 of the rats.

In 3 rats of each group multifiber activity of the saphenous nerve was recorded after producing the extravasation by antidromic stimulation. The innervation area of the saphenous nerve was mapped by scratching the skin with a needle and compared to the area of blue colouration. In all rats of all groups the boundaries of the innervation areas of the saphenous nerve were identical when mapped by electrophysiological methods and the Evans blue dye technique.

The results in the control group and the sciatic nerve cut group are in line with the recent findings made in two other laboratories [1, 4]. Thus, sciatic cut induces a collateral sprouting of $\mathrm{C}$-nociceptive fibers of the saphenous nerve into the denervated area. However, one study reported no sprouting of nociceptive C-fibers after the neighbouring cutaneous nerve was cut [10]. The reason for this discrepancy is not known but among the possible explanations are the following. When the area of extravasation is determined by measuring the Evans blue dye content in an excised piece of skin [10], the sprouting effect found may be less than when simply determining the boundaries of coloured area (the current study) [1, 4]; the colouration is sparser in the area with sprouted axons [4] which may lead to underestimation of the spread of sprouting axons when determined with photometric methods from excised skin flaps [10]. Sprouting of nociceptive C-fibers reaches its maximum by 24 days [4], and at longer survival times the area of sprouting may even become smaller [13]; in the study with negative findings concerning the sprouting of nociceptive Cfibers the measurements were made 240 days after the nerve lesion [10], whereas the studies with positive findings had shorter survival times (the current study) $[1,4]$. The age of the animals tested also greatly influences the spread of sprouting [13]. Furthermore, it has been demonstrated that skin stimulation promotes sprouting (ref. 16; however, see ref. 6). Environmental conditions in some laboratories may be more stressful (e.g. cold) than in others which may increase self-mutilation and promote sprouting. 
Capsaicin treatment of the sciatic nerve to lesion the $\mathrm{C}$-fibers did not produce any collateral sprouting of nociceptive C-fibers of the saphenous nerve. However, as shown previously, and in this study, the local application of capsaicin did at least functionally impair the nociceptive C-fibers of the sciatic nerve as early as $15 \mathrm{~min}$ after the end of the application [18] and this effect lasted at least up to one month [11]. Consistently, it has been reported that capsaicin applied to the nerve trunk in adult animals produces long-term morphological loss of C-fibers [15] although some discrepancy concerning the morphological effects still exists in the literature [2]. These findings suggest that the existence of normal myelinated fibers and/or functionally impaired nociceptive C-fibers is enough to prevent collateral sprouting of the nociceptive $\mathrm{C}$-fibers from the neighboring intact nerve.

Electrophysiological and dye-labelled extravasation methods gave coincident results concerning the innervation area of the saphenous nerve in all experimental conditions. When recording multifiber activity, it is practically not from C-fibers. Thus, the electrophysiological activity evoked from the expanded area suggests that Afibers sprout at least at the same pace as C-fibers. However, the number of sprouted thin myelinated fibers outside the dye-labelled area may be too small to be detected with this crude electrophysiological method.

The present results support the evidence indicating that recovery of pain sensibility after a peripheral nerve lesion may in some cases be explained by sprouting of neighbouring intact nerves to the denervated area. It remains to be studied whether sprouted nociceptive axons have properties which would contribute to the various pain states often followed by peripheral nerve lesions, e.g. whether sprouted nociceptive axons are spontaneously active or whether they have lowered thresholds, is not yet known.

At the time of this study the author had a NIH Fogarty Fellowship (FO5 TW03798-01).

1 Brenan, A., Collateral reinnervation of skin by C-fibres following nerve injury in the rat, Brain Res., 385 (1986) 152-155.

2 Buck, S.H. and Burks, T.F., The neuropharmacology of capsaicin: review of some recent observations, Pharmacol. Rev., 38 (1986) 179-226.

3 Devor, M., Schonfeld, S., Seltzer, Z. and Wall, P.D., Two modes of cutaneous reinnervation following peripheral nerve injury, J. Comp. Neurol., 185 (1979) $211-220$.

4 Doucette, R. and Diamond, J., Normal and precocious sprouting of heat nociceptors in the skin of adult rats, J. Comp. Neurol., 261 (1987) 592-603.

5 Fitzgerald, M., Capsaicin and sensory neurones - a review, Pain, 15 (1983) 109-130.

6 Greenfield, Z. and Devor, M., Collateral reinnervation of rat hindlimb skin does not depend on repeated sensory testing, Neurosci. Lett., 25 (1981) 305-309.

7 Hoffert, M.J., Greenberg, R.P., Wolskee, P.J., Gracely, R.H., Wirdzek, P.R., Vinayakom, K. and Dubner, R., Abnormal and collateral innervations of sympathetic and peripheral sensory fields associated with a case of causalgia, Pain, 20 (1984) $1-12$.

8 Horch, K., Absence of functional collateral sprouting of mechanoreceptor axons into denervated areas of mammalian skin, Exp. Neurol., 74 (1981) 313-317.

9 Inbal, R., Rousso, M., Ashur, H., Wall, P.D. and Devor, M., Collateral sprouting in skin and sensory recovery after nerve injury in man, Pain, 28 (1987) 141-154. 
10 Jansco, G. and Kiraly, E., Cutaneous nerve regeneration in the rat: reinnervation of the denervated skin by regenerative but not by collateral sprouting, Neurosci. Lett., 36 (1983) 133-137.

11 Jansco, G., Kiraly, E. and Jansco-Gabor, A., Direct evidence for an axonal site of action of capsaicin, Naunyn-Schmiedebergs Arch. Pharmacol., 313 (1980) 91-94.

12 Kenins, P., Identification of the unmyelinated sensory nerves which evoke plasma extravasation in response to antidromic stimulation, Neurosci. Lett., 25 (1981) 137-141.

13 Kinnman, E. and Aldskogius, H., Collateral sprouting of sensory axons in the glabrous skin of the hindpaw after chronic sciatic lesion in adult and neonatal rats: a morphological study, Brain Res., 377 (1986) 73-82.

14 Livingston, W.K., Evidence of active invasion of denervated areas by sensory fibers from neighbouring nerves in man, J. Neurosurg., 4 (1947) 140-145.

15 Lynn, B. and Pini, A., Long-term block of afferent C-fibres following capsaicin treatment in the rat, J. Physiol. (Lond.), 362 (1985) 19P.

16 Nixon, B.J., Doucette, R., Jackson, P.C. and Diamond, J., Impulse activity evoked precocious sprouting of nociceptive nerves into dennervated skin, Somatosens. Res., 2 (1984) 97-126.

17 Nurmikko, T. and Pertovaara, A., Painful hyperaesthesia following resection of the lateral cutaneous nerve of the thigh, J. Neurol. Neurosurg. Psychiatry, 47 (1984) 320-321.

18 Petsche, U., Fleischer, E., Lembeck, F. and Handwerker, H.O., The effect of capsaicin application to a peripheral nerve impulse conduction in functionally identified afferent nerve fibers, Brain Res., 265 (1983) 233-240.

19 Weddell, G., Guttman, L. and Guttman, E., The local extensions of nerve fibers into denervated areas of the skin, J. Neurol. Psychiatry, 4 (1941) 206-225. 